\title{
Histological subtypes of symptomatic central nervous system tumours in Singapore
}

\author{
A Das, C A T Chapman, W M Yap
}

\begin{abstract}
The objective was to identify the different subtypes of symptomatic CNS tumours that are encountered in Singapore. Our hospital pathology and operative records from 1994 to 1998 were reviewed and information regarding all patients who underwent biopsy or resection as part of their diagnostic and therapeutic evaluation was extracted. Only histologically confirmed tumours were included in this analysis. Meningiomas made up the largest subgroup of tumours, accounting for $35.1 \%$ of all tumours. In order of decreasing frequency, the remaining most often reported histologies were pituitary adenomas $(11.8 \%)$, secondary neoplasms $(10 \%)$, tumours of nerve sheath $(\mathbf{9 . 4 \%})$, glioblastoma multiforme $(9.3 \%)$, astrocytomas including anaplastic, diffuse and pilocytic (9.2\%), primary CNS lymphomas $(2.9 \%)$, oligodendrogliomas $(2.2 \%)$, hemangioblastomas (2.2\%), craniopharyngiomas $(1.7 \%)$, and embryonal tumours $(1.2 \%)$. Genetic and environmental factors may be responsible for the proportionately higher than expected percentage of meningiomas seen and further study is required to identify these factors.

(F Neurol Neurosurg Psychiatry 2000;68:372-374)
\end{abstract}

Department of

Neurology, National

Neuroscience Institute, Singapore

A Das

Department of Clinical Epidemiology, Tan Tock Seng Hospital, Singapore

C A T Chapman

Department of Pathology WM Yap

Correspondence to: Dr Asha Das, Department of Neurology, National Neuroscience Institute,

11 Jalan Tan Tock Seng,

Singapore 308443

email

Asha_Das@notes.ttsh.gov.sg

Received 29 June 1999 and in final form

4 October 1999

Accepted 5 October 1999
Keywords: central nervous system tumours; Singapore; southeast Asia

Characterising the different forms and range of CNS neoplasms in different regions may provide aetiological clues to some tumour types. Histological descriptions of CNS tumours as they occur in south east Asia and, in particular, in Singapore are rare. One of the few such studies in Singapore by the National Cancer Registry, which describes trends in cancer incidence from 1968 to 1992 , contains information regarding only 594 malignant brain and nervous system tumours over this lengthy period. ${ }^{1}$ The limitations of using a cancer registry, particularly the underepresentation of benign tumours, is immediately apparent. A more recent survey was required to document the current trends in relative frequencies of symptomatic CNS tumours in Singapore. In part these investigations on the relative incidence, age, and sex of patients with tumours of the CNS are important in providing potential aetiological clues to pathogenesis. Certain cultural and religious beliefs make necropsy studies extremely difficult in Singapore. Given such constraints, our study represents the largest and most recent compilation of data on CNS tumours in Singapore.

\section{Methods}

This study was undertaken at a government hospital, which has the largest neurosurgical unit in Singapore, performing 1200 procedures annually. This hospital serves a predominantly adult patient population within a catchment area of 1 million people. As there are no other hospitals within the vicinity, the distribution of cases is unlikely to be biased by physicians' subspecialty interests. As part of a retrospective survey, 48001 pathology reports and the neurosurgical records from 1 January 1994 to 31 December 1998 were reviewed and all histologically confirmed CNS tumours were included in this analysis. None of the histopathological data were obtained from necropsy series; all patients underwent biopsy or resection as part of their diagnostic or therapeutic evaluation.

Age, sex, ethnic background, date of diagnosis, and histological diagnosis were recorded for all patients with CNS tumours. The date of diagnosis is the date on which the pathological diagnosis was obtained. All tumours covered by the World Health Organisation (WHO) classification scheme except for cysts were included. ${ }^{2}$ Tumours diagnosed on the basis of neuroimaging studies without histological confirmation were excluded.

\section{Results}

During a 5 year period from 1 January 1994 to 31 December 1998, 655 tumours of the CNS were diagnosed and, of this total number, 583 were newly diagnosed. We report only on the 583 newly diagnosed tumours. The remaining 72 were recurrent tumours that occurred in the cohort of 583 patients. No patient had two different CNS neoplasms. The ethnic origin of the 583 patients including Chinese $(80.8 \%)$, Malays $(11.5 \%)$, Indians $(5.0 \%)$, and other groups $(2.7 \%)$ reflected the percentage distribution of Singapore's population. ${ }^{3}$ Patients included 307 women (52.7\%) and 276 men 
Table 1 Distribution of CNS tumours by histology and sex

\begin{tabular}{|c|c|c|c|}
\hline & Males & Females & Total (\%) \\
\hline \multicolumn{4}{|l|}{ Tumours of neuroepithelial tissue: } \\
\hline \multicolumn{4}{|l|}{ Astrocytic tumours } \\
\hline Pilocytic astrocytoma (WHO grade I) & 11 & 8 & $19(3.3)$ \\
\hline Astrocytoma (WHO grade II) & 9 & 5 & $14(2.4)$ \\
\hline Anaplastic astrocytoma (WHO grade III) & 7 & 12 & $19(3.3)$ \\
\hline Glioblastoma (WHO grade IV) & 31 & 23 & $54(9.3)$ \\
\hline Subependymal giant cell astrocytoma & 1 & & $1(0.2)$ \\
\hline \multicolumn{4}{|l|}{ Oligodendroglial tumours } \\
\hline Oligodendroglioma & 7 & 3 & $10(1.7)$ \\
\hline Anaplastic oligodendroglioma & 2 & 1 & $3(0.5)$ \\
\hline Ependymal tumours & 1 & 4 & $5(0.9)$ \\
\hline Mixed gliomas & 3 & & $3(0.5)$ \\
\hline \multicolumn{4}{|l|}{ Neuroepithelial tumours of uncertain origin } \\
\hline Gliomatosis cerebri & 1 & & $1(0.2)$ \\
\hline \multicolumn{4}{|l|}{ Choroid plexus tumours } \\
\hline Choroid plexus papilloma & 1 & 2 & $3(0.5)$ \\
\hline \multicolumn{4}{|l|}{ Neuronal and mixed neuronal-glial tumours } \\
\hline Ganglioma & 1 & 1 & $2(0.3)$ \\
\hline Central neurocytoma & 1 & 1 & $2(0.3)$ \\
\hline \multicolumn{4}{|c|}{ Tumours with neuroblastic or glioblastic elements (embryonal tumours) } \\
\hline Primitive neuroectodermal & 1 & 3 & $4(0.7)$ \\
\hline Neuroblastoma & 2 & 1 & $3(0.5)$ \\
\hline \multicolumn{4}{|l|}{ Tumours of the sellar region } \\
\hline Pituitary adenoma & 45 & 24 & $69(11.8)$ \\
\hline Craniopharyngioma & 6 & 4 & $10(1.7)$ \\
\hline \multicolumn{4}{|l|}{ Haematopoietic tumours } \\
\hline \multicolumn{4}{|l|}{ Lymphoma } \\
\hline B cell & 9 & 6 & $15(2.6)$ \\
\hline $\mathrm{T}$ cell & 1 & 1 & $2(0.3)$ \\
\hline \multicolumn{4}{|l|}{ Germ cell tumours } \\
\hline Germinoma & 3 & 1 & $4(0.7)$ \\
\hline Teratoma & 2 & & $2(0.3)$ \\
\hline \multicolumn{4}{|l|}{ Tumours of the meninges } \\
\hline Benign meningioma & 52 & 133 & $185(31.7)$ \\
\hline Atypical meningioma & 6 & 8 & $14(2.4)$ \\
\hline Malignant meningioma & 3 & 3 & $6(1.0)$ \\
\hline \multicolumn{4}{|l|}{ Non-meningothelial tumours of the meninges } \\
\hline \multicolumn{4}{|l|}{ Malignant mesenchymal } \\
\hline Hemangiopericytoma & & 4 & $4(0.7)$ \\
\hline \multicolumn{4}{|l|}{ Tumours of uncertain histogenesis } \\
\hline Haemangioblastoma & 4 & 9 & $13(2.2)$ \\
\hline Tumours of cranial and spinal nerves & 28 & 27 & $55(9.4)$ \\
\hline \multicolumn{4}{|l|}{ Local extensions from regional tumours } \\
\hline Paraganglioma & & 1 & $1(0.2)$ \\
\hline Chordoma & 1 & & $1(0.2)$ \\
\hline \multicolumn{4}{|l|}{ Metastatic neoplasms } \\
\hline Adenocarcinoma & 20 & 13 & $33(5.7)$ \\
\hline Squamous cell carcinoma & 2 & & $2(0.3)$ \\
\hline Metastatic carcinoma & $1 \overline{5}$ & 8 & $23(4.0)$ \\
\hline \multicolumn{4}{|l|}{ Unclassified tumours } \\
\hline Haemangioendothelioma & & 1 & $1(0.2)$ \\
\hline Total & 276 & 307 & $583(100)$ \\
\hline
\end{tabular}

$(47.3 \%)$ giving a male:female ratio of $1: 1.1$. Data on the histology of symptomatic newly diagnosed CNS tumours with pathological verification are outlined in the table.

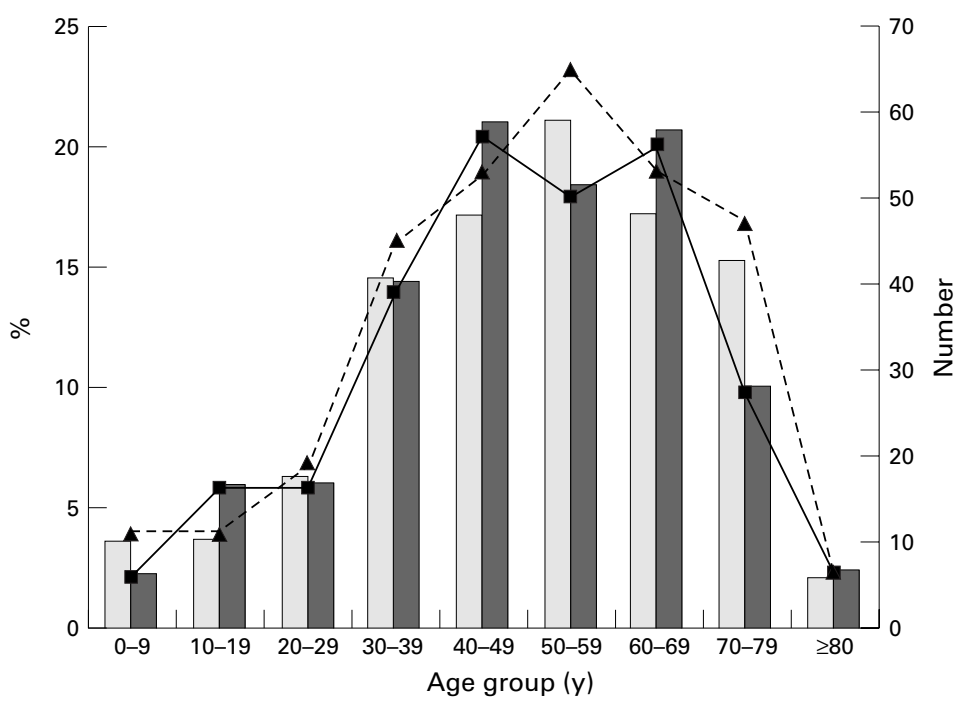

Age distribution by sex. Light bars \% women; dark bars \% men; triangles number of women; squares number of men.
The most commonly encountered tumours were meningiomas, which comprised $35.1 \%$ of all newly diagnosed tumours in our series with $9.8 \%$ of those initially diagnosed being of the atypical or malignant variety. Atypical meningiomas demonstrated hypercellularity, patternless or sheet-like growth, and brisk mitotic activity, and malignant meningiomas demonstrated brain invasion. The second largest group of 69 cases $(11.8 \%)$ were pituitary adenomas. In order of decreasing frequency, the remaining most often reported histologies were secondary neoplasms $(10 \%)$, tumours of the nerve sheath $(9.4 \%)$, glioblastoma multiforme $(9.3 \%)$, astrocytomas including anaplastic, diffuse, and pilocytic $(9.2 \%)$, primary CNS lymphomas $(2.9 \%)$, oligodendrogliomas $(2.2 \%)$, haemangioblastomas $(2.2 \%)$, craniopharyngiomas $(1.7 \%)$, embryonal tumours $(1.2 \%)$, germ cell tumours $(1.0 \%)$, ependymomas $(0.9 \%)$, neuronal tumours $(0.6 \%)$, choroid plexus tumours $(0.5 \%)$, and chordomas $(0.1 \%)$. When histological subtypes were categorised according to ethnicity, no significant differences in the distribution of tumour subtypes among the different ethnic groups were seen.

Females had a higher incidence of benign meningiomas than males, with a male to female ratio of $1: 2.3$ ( $p>0.001)$. However, no significant differences in sex predominance were noted for either the atypical or malignant meningiomas. Although neuroepithelial and pituitary tumours occurred slightly more often in men than women, this difference was again not significant.

The median age at presentation was 51 years (range 0.58 to 87 years) with no significant difference between men and women $(p=0.330)$. This cohort's age distribution is outlined in the figure. The incidence of CNS tumours increased from the 4 th to the 7 th decade. Of the various tumour types progressively higher grade astrocytic tumours presented in progressively older patients with median ages of 20.0 years for grade I astrocytomas, 31.5 years for grade II astrocytomas, 34.5 years for grade III astrocytomas, and 55 years for grade IV astrocytomas.

\section{Discussion}

Our report examines the different subtypes of all symptomatic and pathologically proved CNS neoplasms at a single institution, reflecting the selection bias that is inherent in any hospital series. We estimate that our cohort of 655 tumours from 583 patients includes $50 \%$ of all CNS tumours diagnosed in this country over a 5 year period from 1994 to 1998. Previously, differences in histological subtypes among ethnic groups living in the same geographical region have implicated racial factors in the pathogenesis of CNS tumours. ${ }^{4}$ The problematic classification of numerous histological categories and lack of consensus on diagnostic criteria complicates the comparison of tumour incidence across regions. ${ }^{5}$ Nevertheless, we compared our series with others in an attempt to ascertain if unique trends in histological subtypes were present in this part of south east Asia. Whereas astrocyto- 
mas are the most common primary tumour type in other series from Japan, Thailand, United States, and the previous USSR, ${ }^{6}$ meningiomas seem to be proportionately more common in our series. In population based studies from the west, neuroepithelial tumours account for $40 \%$ to $67 \%$ and meningiomas for $9 \%$ to $27 \%$ of CNS tumours ${ }^{7}$ compared with respective rates of $24.5 \%$ and $35.1 \%$ in our series. This finding is consistent with several reports of non-white ethnic groups showing higher rates of meningiomas, including males from Asia and the Middle East residing in Victoria, Australia, ${ }^{8}$ African-Americans, ${ }^{9}$ and series from China ${ }^{10}$ and Hong Kong. ${ }^{11}$ In many series only $5 \%$ of meningiomas are atypical or malignant, whereas in our series $9.8 \%$ are atypical or malignant. ${ }^{12}$ These findings are likely to be explained at least in part by genetic factors. Tumours of the pituitary and tumours of the cranial and spinal nerves which account for $11.8 \%$ and $9.4 \%$ of reported histologies were more common than other series, in which these tumours account for $8 \%$ and $6.5 \%$ of tumour types. ${ }^{13}$ Tumours diagnosed by neuroimaging but without histological confirmation were excluded. This exclusion might produce a pathological bias in that tumours of the cranial nerves detected on neuroimaging studies are often treated without biopsy. As the Singapore Cancer Registry does not record the incidence rates for benign brain tumours, the population incidence rates cannot be provided. We cannot conclude that the increased proportion of certain tumour subtypes found in our series reflects an increased population incidence rate for meningiomas in Singapore. To really explore such incidence rates will require further examination in future cancer registry studies.

Brain tumours are thought to occur more often in males than in females. ${ }^{7}$ A comparison using international data of the incidence of primary intracranial tumours of all histological types results in an average male to female ratio of 1.4:1 (range 0.9-2.6) across geographical areas. ${ }^{14}$ However, the sex ratio varies considerably by histological type. Gliomas are higher in males with a male to female ratio of $1.5: 1$ and meningiomas are higher in females with a sex ratio of $0.65 .^{15}$ The lower male to female ratio of $1: 1.1$ in our study may be due to the female preponderance of meningiomas. Reports on pituitary adenomas from the United Kingdom show a constant and sizeable predominance of females for all ages and especially among women of childbearing age. ${ }^{16}$ By contrast, in our series males had a higher predominance of pituitary adenomas with a male to female ratio of 1.88:1. This finding is surprising and the reason for this male predominance of pituitary adenomas is difficult to explain.

Our series of symptomatic brain tumours inclusive of all histologically confirmed tumours over a 5 year period highlights a preponderance of meningiomas. Higher than expected percentages of reported brain tumours include tumours of cranial and spinal nerves and pituitary tumours. Glioblastoma multiforme occurred less often. Genetic and environmental factors may be responsible for our histological findings and further study is required to identify these factors.

We thank Mr Chan Siew Pang (GIMA) from the Department of Clinical Epidemiology for statistical assistance.

1 Chia KS, Lee HP, Seow A, et al. Trends in cancer incidence in Singapore 1968-1992. 1996;112.

2 Kleihues P, Burger PC, Scheithauer BW. The new WHO Kleihues P, Burger PC, Scheithauer BW. The new WHO
classification of brain tumours. Brain Pathology 1993;3: 255-68.

3 Singapore Population Census Reports 1970, 1980, 1990. Singapore: Department of Statistics, 1990

4 Velema JP, Percy CL. Age curves of central nervous system tumor incidence in adults: variation of shape in histologic type. F Natl Cancer Inst 1987;79:623-9.

5 Zülch KJ. Histological typing of tumors of the central nervous system. International histological classification of tumors (21). Geneva: World Health Organization, 1979.

6 Schiffer D. Descriptive epidemiology of primary nervous tumours in brain tumors 2nd ed. New York: Springer-Verlag: Berlin, 1997:132-6.

7 Huang WQ, Zheng SJ, Tian QS, et al. Statistical analysis of central nervous system tumors in China. F Neurosurg 1982; 56:555-64.

8 Takeshita I, Yang GR, Piao HZ, et al. Comparative study of brain tumors treated at China Medical University, China, brain tumors treated at China Medical University, China, 83:386-91.

9 Fan KJ, Pezeshkpour GH. Ethnic distribution of primary central nervous system tumors in Washington, DC, 1971 to 1985. F Natl Med Assoc 1992;84:858-63.

$10 \mathrm{Ng}$ HK, Poon WS, South JR, et al. Tumours of the central nervous system in Chinese in Hong Kong: a histological review. Aust NZ F Surg 1988;58:573-8.

11 Fan KJ, Kovi J, Earle KM. The ethnic distribution of primary central nervous system tumors: AFIP, 1958-70. $\mathcal{F}$ Neuropathol Exp Neurol 1977;36:41-9.

12 Preston-Martin S, Staples M, Farrugia H, et al. Primary tumors of the brain, cranial nerves, and cranial meninges in Victoria, Australia, 1982-90: pattern of incidence and survival. Neuroepidemiology 1993;12:270-9.

13 Surawicz TS, McCarthy BJ, Kupelian V, et al. Descriptive epidemiology of primary brain and CNS tumors: results from the Central Brain Tumor Registry of the United from the Central Brain Tumor Registry of

14 Heshmat MY, Kovi J, Simpson C, et al. Neoplasms of the central nervous system. Incidence and population selectivity in the Washington DC, Metropolitan area. Cancer 1976; 38:2135-42.

15 Velema JP, Walker AM. The age curve of nervous system tumor incidence in adults: common shape but changing levels by sex, race, and geographic location. Int $\mathcal{F}$ Epidemiol 1987;16:177-83.

16 Robinson N, Beral V, Ashley JSA. Incidence of pituitary adenoma in women. Lancet 1979;ii:630. 